Article

\title{
Energy Intake from Healthy Foods Is Associated with Motor Fitness in Addition to Physical Activity: A Cross-Sectional Study of First-Grade Schoolchildren in Japan
}

\author{
Naoko Hatta ${ }^{1}$, Yuki Tada ${ }^{2, *(\mathbb{D}}$, Kazuko Ishikawa-Takata ${ }^{2} \mathbb{D}$, Tadasu Furusho ${ }^{3}$, Rieko Kanehara ${ }^{1} \mathbb{D}$, Toshiki Hata ${ }^{1}$, \\ Azumi Hida ${ }^{2}$ (D) and Yukari Kawano 2 (D) \\ 1 Graduate School of Agriculture, Tokyo University of Agriculture, Sakuragaoka 1-1-1, Setagaya, \\ Tokyo 156-8502, Japan; nyako.705@gmail.com (N.H.); riekokk07@gmail.com (R.K.); \\ 11320001@nodai.ac.jp (T.H.) \\ 2 Department of Nutritional Science, Faculty of Applied Bioscience, Tokyo University of Agriculture, \\ Sakuragaoka 1-1-1, Setagaya, Tokyo 156-8502, Japan; kt207460@nodai.ac.jp (K.I.-T.); \\ a3hida@nodai.ac.jp (A.H.); y1kawano@nodai.ac.jp (Y.K.) \\ 3 Department of International Food and Agricultural Science, Faculty of International Agriculture and Food \\ Studies, Tokyo University of Agriculture, Sakuragaoka 1-1-1, Setagaya, Tokyo 156-8502, Japan; \\ tfurusho@nodai.ac.jp \\ * Correspondence: y3tada@nodai.ac.jp; Tel.: +81-(0)3-5477-2670
}

Citation: Hatta, N.; Tada, Y.; Ishikawa-Takata, K.; Furusho, T.; Kanehara, R.; Hata, T.; Hida, A.; Kawano, Y. Energy Intake from Healthy Foods Is Associated with Motor Fitness in Addition to Physical Activity: A Cross-Sectional Study of First-Grade Schoolchildren in Japan. Int. J. Environ. Res. Public Health 2022, 19, 1819. https://doi.org/10.3390/ ijerph19031819

Academic Editor: Paul B. Tchounwou

Received: 6 January 2022

Accepted: 2 February 2022

Published: 5 February 2022

Publisher's Note: MDPI stays neutral with regard to jurisdictional claims in published maps and institutional affiliations.

Copyright: (C) 2022 by the authors. Licensee MDPI, Basel, Switzerland. This article is an open access article distributed under the terms and conditions of the Creative Commons Attribution (CC BY) license (https:// creativecommons.org/licenses/by/ $4.0 /)$

\begin{abstract}
Childhood motor fitness is important for the physical and mental health of children and the prevention of future lifestyle diseases. This study aimed to investigate how energy intake from healthy foods and physical activity are associated with motor fitness among first-grade children. First-grade children (aged 6-7 years) attending three public elementary schools in Tokyo, Japan $(n=884)$, participated in this cross-sectional study. Self-administered questionnaires were distributed, which focused on lifestyle habits and required completion of a 1-day dietary record of meals that children ate at home. School lunch consumption was also assessed. Motor fitness was assessed by the New Physical Fitness Test (NPFT). Multiple regression analysis was used to investigate the association of the amount of energy from healthy foods and physical activity with NPFT scores. NPFT scores were positively correlated with involvement in after school exercise classes, playing outside (in boys only), and total energy intake. Energy intake from healthy foods showed a positively associated with NPFT scores (boys, $\beta=0.120, p=0.011$; girls, $\beta=0.140, p=0.004$ ), while energy intake from unhealthy foods did not. Energy intake from healthy foods was associated with motor fitness in children in addition to physical activity.
\end{abstract}

Keywords: children; motor fitness; diet; lifestyle habit

\section{Introduction}

Previous studies have found that cardiorespiratory endurance and muscular strength in childhood and adolescence are associated with systemic and abdominal fat, bone composition, and mental health [1]. García-Hermoso reviewed studies examining the association between muscular fitness during childhood and health parameters later in life and concluded that having high fitness reduced anthropometric and adiposity parameters, insulin resistance, and triglycerides [2]. Higher childhood motor fitness is important not only for the physical and mental health of children, but also for the prevention of future lifestyle diseases; thus, improving children's motor fitness is vital.

The motor fitness of Japanese elementary school children had been growing until around 1985, but has been gradually declining since then, especially in their ability to run, jump and throw. The New Physical Fitness Test (NPFT) is implemented annually to Japanese primary school children, and the Ministry of Education, Culture, Sports, Science and Technology (MEXT) aims to exceed the standard set results of 1985, which indicated a 
record high for children's motor fitness [3]. This reduction in motor fitness is concerning, as it could lead to a lack of exercise and reduced motor fitness in the future.

Physical activity is one of the most important factors for achieving high motor fitness [4]. However, in addition to physical activity, the effect of dietary habits on motor fitness has been suggested [5]. One report indicates that adolescent children who eat breakfast every day have higher cardiorespiratory endurance than do children who skip breakfast [6]. Zaqout et al. [7] also demonstrated that consumption of fruit and vegetables is one of determinants of child's motor fitness in addition to gender, body mass index (BMI), psychological factors, and amount of physical activity. Children in the growth phase must consume sufficient energy to support tissue synthesis and physical activity [8]. Previous studies have also reported that the content of food consumed by children is related to their physical and mental health $[9,10]$. However, most of these previous studies $[6,7,10]$ reported on the consumption frequency of fruits and vegetables, or unhealthy foods, or evaluated the frequency of intake of limited food groups on a semi-quantitative basis, and did not assess whole dietary habit.

We hypothesized that children who receive more energy from healthy food groups have a higher motor fitness level, as well as a higher physical activity level. To test this, we collected nationally standardized NPFT scores and dietary records from first-grade children in Japan. The present study aimed to investigate how healthy foods and physical activity are associated with motor fitness among first-grade children, after adjustment for several confounding factors.

\section{Materials and Methods}

A cross-sectional study of children and their guardians during the first year of compulsory education was conducted.

\subsection{Participants}

Participants were recruited from 1982 pairs of first-grade students aged 6-7 years in three public primary schools in Tokyo and their guardians between April 2013 and April 2018. Responses were obtained from 1018 pairs (51.4\%). Of these, 884 pairs $(51.2 \%$ boys and $48.8 \%$ girls) were analyzed after questionnaires with missing answers were excluded (Figure 1). This study was conducted according to the guidelines outlined in the Declaration of Helsinki. All procedures involving research study participants were approved by the Human Research Ethics Committees at Tokyo University of Agriculture (Reference numbers: 1122, 1130, 1501, 1605, 1704, 1802). Written informed consent was obtained from all participants.

\subsection{Data Collection}

The researcher explained the aim and content of the survey to school principals and class teachers in each school. The class teachers distributed study protocols, consent forms, lifestyle questionnaires, and dietary records of meals that children ate at home. The survey forms were distributed annually between late May and early June, from 2013 to 2018, and were collected two weeks later. Consent forms and questionnaires were filled out at home, placed in an unsigned envelope, and submitted to each school. This study complied with the Strengthening the Reporting of Observational Studies in Epidemiology-nutrition epidemiology (STROBE-nut) guidelines [11]. 


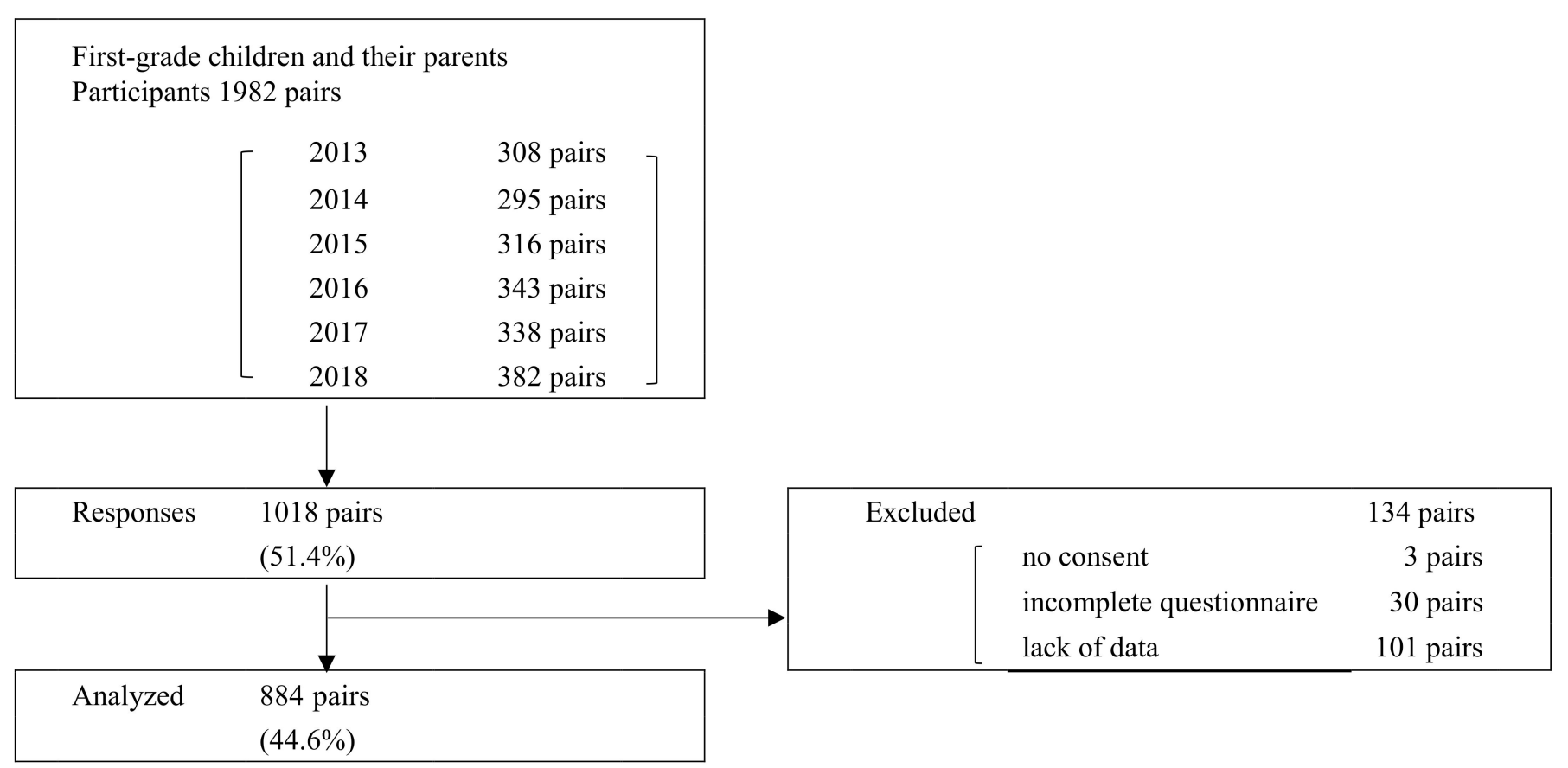

Figure 1. Flowchart of participant selection.

\subsection{Measures}

\subsubsection{Motor Fitness (New Physical Fitness Test Score)}

Motor fitness was assessed during the first term of every school year, based on the MEXT New Physical Fitness Test Implementation Guideline in each school [12]. The following items were measured: grip strength, sit-ups, sit and reach, side-steps, 20-metre shuttle run, 50-metre run, standing long jump, and softball throw (see Supplementary Materials). Elementary school teachers who are familiar with the measurement procedure conducted the test in their physical education classes, and the researchers received the data for the children participating in the study. Each measurement is allocated a score according to sex, in accordance with the Implementation Guideline. The total score for these eight items (NPFT score) was used as an index of motor fitness in the present study.

\subsubsection{Characteristics and Lifestyle Habits of Children}

Participating children and their guardians were instructed to complete a printed questionnaire on characteristics and lifestyle of children at home. Participating children were asked to indicate the frequency of playing outside at break time. Guardians were asked to provide information about the lifestyle of children: waking time; bedtime; frequency of eating breakfast per week; participation in after school classes including weekend classes; duration of watching television, video gaming, or reading comics, and studying per day at home. After school classes were categorised into the following three groups: exercise (e.g., baseball, soccer, swimming, gymnastics, basketball, tennis, or ballet), music (e.g., violin, piano, orchestra, drums, or chorus), and classroom learning [e.g., painting, science, language, juku (cram school), calligraphy, abacus, or religious studies]. Lesson time per week was calculated as the time spent at each session multiplied by the number of times each session is performed per week. Guardians were also asked to record their children's height and weight measured at the health check-up conducted at each elementary school in April. A child's physique was calculated using the following Rohrer's index formula: weight $(\mathrm{kg}) /$ height $(\mathrm{cm})^{3} \times 10^{7}$. 


\subsubsection{Children's Dietary Habits}

(1) Breakfast, dinner, and between-meal eating at home

Guardians filled out a dietary record to provide the information regarding the menu, ingredients, and weights of ingredients (including breakfast, dinner, and between-meal eating at home) for one average school day.

\section{(2) Lunch (school lunch)}

Japanese school lunches are provided every weekday based on school lunch implementation standards [13] in accordance with Dietary Reference Intakes for Japanese [8] set forth by the Ministry of Health, Labour and Welfare. Each school was surveyed for one day with regular menus. Investigators (registered dieticians and students in a nutrition course) observed children from the time they were served each dish up until the point they returned the trays. Consumption rate for each dish was evaluated using five categories ( $0 \%$ (food untouched), 25\%, 50\%, 75\%, and 100\% (completed)). Total intake was calculated based on the nutritional value of each menu and consumption rate for each child.

(3) Nutritional value calculation

Daily intake was calculated by combining intakes of breakfast, dinner, and betweenmeal eating at home with lunch intake. The energy content of surveyed meals was calculated using nutritional value calculation software (Excel Eiyo-kun, Version 8.0), with values based on the 2015 Standard Tables of Food Composition in Japan (Seventh Revised Version) [14]. Food consumed by children was divided into six food groups, according to the criteria in the Japanese Food Guide Spinning Top by the Ministry of Agriculture, Forestry and Fisheries and the Ministry of Health, Labour and Welfare [15], and numbers of servings were calculated. One serving (SV) was defined as the following: $40 \mathrm{~g}$ of carbohydrates for grain dishes; $70 \mathrm{~g}$ of the main ingredient in vegetable dishes; $6 \mathrm{~g}$ of protein in fish or meat dishes; $100 \mathrm{mg}$ of calcium in milk or dairy products; $100 \mathrm{~g}$ of the main ingredient in fruit dishes; or $80 \mathrm{kcal}$ of energy in confectionaries or sweetened beverages.

\section{(4) Breakdown of energy intake}

Energy intake from grain dishes, vegetable dishes, fish and meat dishes, milk and dairy products, and fruit was defined as energy from healthy foods. Energy intake from unhealthy foods was defined as the amount of energy derived from confectionaries and sweetened beverages. Confectionaries were classified according to the 2015 Standard Tables of Food Composition in Japan (Seventh Revised Version) [14]. Although the Standard Tables of Food Composition [14] classified ice cream as a dairy product, this study classified it as a confectionary. Similarly, this study categorized juice beverages that were not $100 \%$ fruit juice or that were from concentrate as sweetened beverages, in accordance with the Japanese Food Guide Spinning Top [15] definition of sweetened beverages.

\subsubsection{Statistical Analysis}

Participant characteristics were compared in relation to NPFT score quintile groups by gender with weighted one-way analyses of variance for continuous variables, and the Mantel-Haenszel test for trend for categorical variables.

A regression analysis adjusted for physique (Rohrer's index) was conducted using the forced entry method for the investigation of items related to NPFT score quintile. When inputting dependant variables, total time spent watching television or participating in video gaming or reading comics was aggregated as sedentary activity at home, and classified into four categories $(<60,60-119,120-179, \geq 180 \mathrm{~min} /$ day). Study time $(<15,15-29,30-44$, $45-59,60-74,75-89,90-104, \geq 105 \mathrm{~min} /$ day $)$, sleep duration $(<8.0,8.0-8.4,8.5-8.9,9.0-9.4$, 9.5-9.9, 10.0-10.4, 10.5-10.9, $\geq 11 \mathrm{~h} /$ day) and Rohrer's index were classified into eight nearly equal groups. Median values in each group were entered as dependent variables. The frequency of outside playing was set as ' 1 ' for responses of "almost every day"; all other responses with a frequency less than "almost every day" were set as ' 0 '. A value of ' 1 ' was allocated for children engaged in after school exercise class, classroom learning, and/or 
music class, whereas children not engaged in after school classes including weekend classes were allocated dummy variables with a value of ' 0 '. A value of ' 1 ' was allocated for a response to the frequency of eating breakfast per week of " 7 times a week", whereas ' 0 ' was allocated for all other responses.

Moderator variables for investigation of the association between NPFT scores and daily energy intake and physical activity included the following: Rohrer's index, sleep duration, time spent studying, sedentary activity at home, participation in after school classes (classroom learning or music), and breakfast eating. As for explanatory variables, in addition to playing outside and participation in after school exercise class, the amount of energy derived from healthy foods was used for model 1. The amount of energy derived from unhealthy foods was used for model 2, and the amounts of energy derived from both healthy foods and unhealthy foods were used for model 3. To demonstrate the appropriateness of using regression analysis, we verified the residuals of model 3 are normality distributed by using the Kolmogorov-Smirnov test. The $\mathrm{G}^{*}$ power 3.1.9.7 program (Faul, F., Erdfelder, E., Buchner, A., \& Lang, A.-G., Heinrich-Heine-Universität Düsseldorf, Germany), the power analysis software, determined the statistical power $(1-\beta)$ as $>0.8$ for the model 3. Data were expressed as means and standard deviations. A $p$ value less than 0.05 was considered statistically significant. Statistical analysis was performed using IBM SPSS Statistics for Windows (Version 27.0).

\section{Results}

\subsection{Characteristics of Participants}

In this study, 453 (51.2\%) of the participating children were boys and $431(48.8 \%)$ were girls. Boys had higher values for height $(p=0.006)$ and weight $(p=0.001)$ than girls, but there was no significant difference in Rohrer's index $(p=0.303)$. There was a sex difference in the results of all measurement items in the NPFT, except for sit-ups ( $p=0.310)$, and boys had higher values in all items except for sit and reach (grip strength, $p=0.011$; other items, $p<0.001$ ). Overall NPFT scores were $30.0 \pm 6.2$ points for boys and $29.5 \pm 6.2$ points for girls, indicating no significant difference $(p=0.252)$.

Comparison of children's characteristics and lifestyle habits in the five NPFT score quintiles indicated that boys with higher NPFT scores had higher heights $(p<0.001)$ and weights $(p<0.001)$. Boys with higher NPFT scores also had longer after school exercise lesson times $(p<0.001)$ and higher frequencies of playing outside $(p<0.001$; Table 1$)$. Girls with higher NPFT scores had longer after school exercise lesson times $(p=0.010)$ and lower Rohrer's index values ( $p=0.039$; Table 2 ).

\subsection{Dietary Intake and New Physical Fitness Test Score}

Boys with higher NPFT scores had higher total energy intake at lunch $(p=0.003)$, total daily energy intake $(p=0.013)$, energy intake derived from healthy foods $(p=0.003)$, vegetable dish intake $(\mathrm{P}=0.001)$, and fish and meat dish intake $(p=0.028$; Table 3$)$. Girls with higher NPFT scores had higher values for total energy intake at breakfast $(p=0.008)$, energy intake from healthy foods at breakfast $(p=0.030)$, total energy intake at lunch $(p<0.001)$, total daily energy intake $(p=0.001)$, energy intake from healthy foods $(p=0.005)$, and fish and meat dish intake ( $p=0.037$; Table 4$)$. 
Table 1. Characteristics and lifestyle habits within New Physical Fitness Test score quintile groups among boys.

\begin{tabular}{|c|c|c|c|c|c|c|c|c|c|c|c|c|c|c|}
\hline \multirow{3}{*}{ Variables } & \multirow{3}{*}{$\begin{array}{l}\text { Median } \\
\text { (Min-Max) }\end{array}$} & \multicolumn{12}{|c|}{ NPFT Score Quintile } & \multirow{3}{*}{$\begin{array}{l}p \text { for } \\
\text { Trend }\end{array}$} \\
\hline & & q1 $(r$ & $n=\varepsilon$ & & \multirow{2}{*}{\multicolumn{3}{|c|}{$\begin{array}{c}\mathrm{q} 2(n=67) \\
26.0 \\
(25.0-27.0)\end{array}$}} & \multirow{2}{*}{\multicolumn{2}{|c|}{$\begin{array}{c}\mathrm{q} 3(n=\widetilde{93}) \\
29.0 \\
(28.0-30.0)\end{array}$}} & \multirow{2}{*}{\multicolumn{2}{|c|}{$\begin{array}{c}\mathrm{q} 4(n=106) \\
32.0 \\
(31.0-34.0)\end{array}$}} & \multirow{2}{*}{\multicolumn{2}{|c|}{$\begin{array}{c}\mathrm{q} 5(n=99) \\
38.0 \\
(35.0-49.0)\end{array}$}} & \\
\hline & & \multicolumn{3}{|c|}{$\begin{array}{c}22.0 \\
(13.0-24.0)\end{array}$} & & & & & & & & & & \\
\hline Height & $(\mathrm{cm})$ & 116.3 & \pm & 5.7 & 117.8 & \pm & 5.5 & 117.6 & \pm 5.1 & $118.8=$ & \pm 4.7 & 119.3 & \pm 4.8 & $<0.001$ \\
\hline Weight & $(\mathrm{m})$ & 20.3 & \pm & 2.7 & 20.7 & \pm & 2.5 & 21.1 & 3.1 & $21.6=$ & 3.2 & 21.5 & \pm 2.6 & $<0.001$ \\
\hline Rohrer's index & $\left(\mathrm{kg} / \mathrm{m}^{3}\right)$ & 129.3 & \pm & 15.0 & 127.1 & \pm & 13.4 & 129.6 & 13.0 & $128.5=$ & 11.5 & 126.8 & \pm 12.9 & 0.335 \\
\hline Grip strength & $\left(1-\frac{1}{2}\right.$ & 6.7 & \pm & 1.9 & 7.8 & \pm & 1.8 & 8.2 & \pm 1.7 & 8.7 & 1.9 & 9.8 & \pm 2.1 & $<0.001$ \\
\hline Sit-ups & & 7.0 & \pm & 4.8 & 9.8 & \pm & 4.6 & 11.5 & \pm 4.5 & 12.7 & \pm 3.1 & 15.6 & \pm 4.6 & 0.001 \\
\hline Sit and reach & m) & 22.1 & \pm & 5.1 & 23.9 & \pm & 6.4 & 24.6 & \pm 6.0 & 26.2 & \pm 5.6 & 29.0 & $\pm \quad 5.5$ & \\
\hline Side-step & points) & 23.2 & \pm & 4.6 & 24.1 & \pm & 5.4 & 26.1 & \pm 4.7 & 28.2 & \pm 4.1 & 31.6 & \pm 3.9 & $<0.001$ \\
\hline 20-metre shuttle run & (times) & 11.9 & \pm & 4.2 & 17.2 & \pm & 7.7 & 17.7 & \pm 7.1 & 22.6 & \pm 11.1 & 28.6 & \pm 13.8 & $<0.001$ \\
\hline 50-me & & 12.3 & \pm & 0.9 & 11.8 & \pm & 0.7 & 11.5 & \pm 0.6 & 10.9 & \pm 0.6 & 10.6 & \pm 0.5 & $<0.001$ \\
\hline Stan & & 97.5 & \pm & 13.5 & 104.4 & \pm & 12.8 & 110.0 & \pm 13.7 & $115.3=$ & 11.3 & 127.3 & \pm 11.7 & $<0.001$ \\
\hline Softball throw & (m) & 5.8 & \pm & 2.2 & 6.1 & \pm & 2.1 & 7.7 & \pm 2.5 & $7.9=$ & \pm 2.7 & 10.5 & \pm 3.5 & $<0.001$ \\
\hline Sleep c & & & \pm & 0.6 & 9.6 & \pm & 0.7 & 9.5 & 0.6 & 9.8 & 0.7 & 9.6 & 0.7 & 0.278 \\
\hline Studying & & 49.1 & \pm & 23.7 & 44.3 & \pm & 19.1 & 42.7 & \pm 21.3 & 47.4 & $\pm \quad 44.7$ & 44.6 & $\pm \quad 21.8$ & 0.529 \\
\hline Watching television & (min/day) & 78.5 & \pm & 61.3 & 74.5 & \pm & 46.4 & 83.8 & $\pm \quad 46.2$ & 70.2 & $\pm \quad 41.0$ & 75.3 & $\pm \quad 42.9$ & 0.442 \\
\hline $\begin{array}{l}\text { Playing video games / } \\
\text { reading comics }\end{array}$ & (min/day) & 20.2 & \pm & 25.3 & 23.2 & \pm & 40.9 & 28.1 & \pm 31.0 & 17.2 & \pm 24.1 & 23.3 & $\pm \quad 23.2$ & 0.973 \\
\hline After school exercise class & (min/week) & 81.8 & \pm & 118.2 & 104.2 & \pm & 107.7 & 114.1 & 126.6 & $155.7=$ & 143.9 & 195.7 & 167.9 & $<0.001$ \\
\hline After school classroom learning & . 1 & 62.7 & \pm & 72.5 & 61.3 & \pm & 79.8 & 45.3 & 80.1 & $47.7=$ & 65.9 & 47.9 & \pm 59.7 & 0.079 \\
\hline After school music class & (min/week) & 9.0 & \pm & 24.2 & 9.6 & \pm & 21.8 & 8.6 & \pm 20.8 & 5.9 & \pm 21.0 & 6.8 & \pm 17.4 & 0.258 \\
\hline Playing outside & 1) & 74 & & t.1) & 61 & $(91$ & 1.0) & 87 & (93.5) & 102 & $(96.2)$ & 95 & $(96.0)$ & $<0.001$ \\
\hline \multirow{4}{*}{ Frequency of eating breakfast } & 0-2 times/week & 0 & $(0$ & & 0 & $(0$. & $.0)$ & 0 & $(0.0)$ & 0 & $(0.0)$ & 0 & $(0.0)$ & \multirow{4}{*}{0.866} \\
\hline & 3-4 times/week & 0 & $(0$ & & 0 & $(0$. & $.0)$ & 0 & $(0.0)$ & 0 & $(0.0)$ & 1 & (1.0) & \\
\hline & 5-6 times/week & 2 & & .3) & 1 & $(1$. & .5) & 2 & (2.2) & 1 & $(0.9)$ & 1 & (1.0) & \\
\hline & 7 times/week & 86 & & 7.7) & 66 & (98 & $3.5)$ & 91 & $(97.8)$ & 105 & (99.1) & 97 & $(98.0)$ & \\
\hline
\end{tabular}

Mean \pm SD or number (\%), $p$ values are based on weighted one-way analysis of variance for continuous variables, or the Mantel-Haenszel test for trend for categorical variables. NPFT, New Physical Fitness Test.

Table 2. Characteristics and lifestyle habits within New Physical Fitness Test score quintile groups among girls.

\begin{tabular}{|c|c|c|c|c|c|c|c|c|c|c|c|c|c|c|c|c|c|}
\hline \multirow{3}{*}{ Variables } & \multirow[b]{3}{*}{$\begin{array}{c}\text { Median } \\
\text { (Min-Max) }\end{array}$} & \multicolumn{15}{|c|}{ NPFT Score Quintile } & \multirow{3}{*}{$\begin{array}{l}p \text { for } \\
\text { Trend }\end{array}$} \\
\hline & & \multicolumn{3}{|c|}{$\mathrm{q} 1(n=70)$} & \multicolumn{3}{|c|}{$\mathrm{q} 2(n=96)$} & \multicolumn{3}{|c|}{$\mathrm{q} 3(n=82)$} & \multicolumn{3}{|c|}{$\mathrm{q} 4(n=91)$} & \multicolumn{3}{|c|}{$\mathrm{q} 5(n=92)$} & \\
\hline & & \multicolumn{3}{|c|}{$\begin{array}{c}21.0 \\
(9.0-23.0) \\
\end{array}$} & \multicolumn{3}{|c|}{$\begin{array}{c}26.0 \\
(24.0-27.0) \\
\end{array}$} & \multicolumn{3}{|c|}{$\begin{array}{c}29.0 \\
(28.0-30.0)\end{array}$} & \multicolumn{3}{|c|}{$\begin{array}{c}33.0 \\
(31.0-34.0)\end{array}$} & \multicolumn{3}{|c|}{$\begin{array}{c}38.0 \\
(35.0-46.0)\end{array}$} & \\
\hline Height & $(\mathrm{cm})$ & 114.2 & \pm & 5.5 & 115.1 & \pm & 5.4 & 117.5 & \pm & 4.6 & 117.7 & \pm & 4.4 & 118.5 & \pm & 4.7 & $<0.001$ \\
\hline Weight & (m) & 19.5 & \pm & 2.9 & 20.0 & \pm & 3.0 & 20.9 & \pm & 3.1 & 20.9 & \pm & 2.9 & 21.3 & \pm & 2.9 & $<0.001$ \\
\hline Rohrer's index & $\left(\mathrm{kg} / \mathrm{m}^{3}\right)$ & 131.0 & \pm & 13.8 & 131.2 & \pm & 14.5 & 128.2 & \pm & 13.4 & 127.8 & \pm & 12.1 & 128.0 & \pm & 13.0 & 0.039 \\
\hline Grip strength & $(\mathrm{kr})$ & 6.3 & \pm & 2.1 & 7.4 & \pm & 1.6 & 7.7 & \pm & 1.5 & 8.4 & \pm & 2.0 & 9.6 & \pm & 2.1 & $<0.001$ \\
\hline Sit-u & mes & 6.5 & \pm & 5.1 & 10.0 & \pm & 5.1 & 11.1 & \pm & 4.2 & 12.4 & \pm & 4.2 & 14.9 & \pm & 3.6 & \\
\hline d reach & m) & 22.9 & \pm & 6.4 & 27.0 & \pm & 6.2 & 28.5 & \pm & 5.3 & 30.0 & \pm & 6.5 & 32.8 & \pm & 6.5 & 0.001 \\
\hline Side-step & (points) & 22.2 & \pm & 6.5 & 24.0 & \pm & 4.8 & 25.7 & \pm & 3.9 & 27.1 & \pm & 3.3 & 28.3 & \pm & 3.9 & $<0.001$ \\
\hline 20-metre shuttle run & (times) & 11.0 & \pm & 5.2 & 14.4 & \pm & 6.6 & 15.6 & \pm & 6.7 & 18.7 & \pm & 8.1 & 23.4 & \pm & 9.4 & $<0.001$ \\
\hline 50-me & (s) & 12.7 & \pm & 1.9 & 12.1 & \pm & 0.7 & 11.6 & \pm & 0.6 & 11.3 & \pm & 0.7 & 10.8 & \pm & 0.5 & $<0.001$ \\
\hline Stan & $(\mathrm{cm})$ & 90.7 & \pm & 20.9 & 99.5 & \pm & 13.3 & 103.8 & \pm & 11.5 & 108.2 & \pm & 12.6 & 118.4 & \pm & 11.5 & $<0.001$ \\
\hline Softball throw & $(\mathrm{m})$ & 3.9 & \pm & 1.5 & 4.4 & \pm & 1.5 & 5.2 & \pm & 1.6 & 5.8 & \pm & 1.6 & 6.4 & \pm & 1.9 & $<0.001$ \\
\hline Sleep & & 9.5 & \pm & 0.7 & 9.7 & \pm & 0.6 & 9.5 & \pm & 0.6 & 9.6 & \pm & 0.5 & 9.6 & \pm & 0.7 & 0.666 \\
\hline Studying & & 41.9 & \pm & 19.9 & 48.9 & \pm & 25.0 & 46.9 & \pm & 34.3 & 41.2 & \pm & 17.4 & 44.8 & \pm & 32.7 & 0.679 \\
\hline Watching television & (min/day) & 64.3 & \pm & 41.6 & 74.2 & \pm & 45.0 & 72.5 & \pm & 48.2 & 66.8 & \pm & 42.9 & 66.0 & \pm & 49.2 & 0.654 \\
\hline $\begin{array}{l}\text { Playing video games/ } \\
\text { reading comics }\end{array}$ & (min/day) & 15.0 & \pm & 25.4 & 14.8 & \pm & 27.5 & 15.3 & \pm & 28.7 & 12.9 & \pm & 20.8 & 12.4 & \pm & 23.2 & 0.397 \\
\hline After school exercise class & $(\min$ & 54.3 & \pm & 61.1 & 73.4 & \pm & 84.6 & 93.9 & \pm & 106.3 & 82.3 & \pm & 90.1 & 96.1 & \pm & 131.4 & 0.010 \\
\hline After school classroom learning & $\min /$ wee & 47.0 & \pm & 78.8 & 59.3 & \pm & 77.6 & 61.2 & \pm & 66.4 & 56.7 & \pm & 91.1 & 39.5 & \pm & 60.1 & 0.379 \\
\hline After school music class & (min/week) & 16.1 & \pm & 30.2 & 22.5 & \pm & 38.6 & 24.8 & & 32.7 & 27.9 & \pm & 36.5 & 23.2 & \pm & 35.9 & 0.145 \\
\hline Playing outside & Always & 60 & $(85$ & .7) & 83 & $(86$ & 6.5) & 73 & 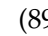 & $9.0)$ & 86 & $(94$ & 4.5) & 84 & $(91$ & 1.3) & 0.069 \\
\hline & $0-2$ times/week & 0 & $(0$ & .0) & 0 & $(0$. & & 0 & $(0$ & $.0)$ & 0 & $(0$. & $.0)$ & 0 & $(0$. & & \\
\hline & 3-4 times/week & 1 & $(1$. & .4) & 1 & $(1$. & & 1 & $(1$ & .2) & 0 & $(0$ & 0) & 0 & $(0$. & & \\
\hline Frequency or eatming oreakrast & 5-6 times/week & 0 & $(0$. & .0) & 3 & (3. & & 3 & $(3$ & .7) & 3 & & $3)$ & 1 & $(1$. & & 0.397 \\
\hline & 7 times/week & 69 & $(98$ & 3.6) & 92 & (95 & 5.8) & 78 & $(95$ & 5.1) & 88 & & 6.7) & 91 & $(98$ & 8.9) & \\
\hline
\end{tabular}

Mean \pm SD or number (\%), $p$ values are based on weighted one-way analysis of variance for continuous variables, or the Mantel-Haenszel test for trend for categorical variables. NPFT, New Physical Fitness Test. 
Table 3. Dietary intake within New Physical Fitness Test score quintile groups among boys.

\begin{tabular}{|c|c|c|c|c|c|c|c|c|c|c|c|c|c|c|c|c|c|}
\hline \multirow{3}{*}{$\begin{array}{l}\text { Variables } \\
\text { Breakfast }\end{array}$} & & \multicolumn{15}{|c|}{ NPFT Score Quintile } & \multirow{2}{*}{$\begin{array}{l}p \text { for } \\
\text { Trend }\end{array}$} \\
\hline & & \multicolumn{3}{|c|}{$\mathrm{q} 1(n=88)$} & \multicolumn{3}{|c|}{$\mathrm{q} 2(n=67)$} & \multicolumn{3}{|c|}{ q3 $(n=93)$} & \multicolumn{3}{|c|}{$\mathrm{q} 4(n=106)$} & \multicolumn{3}{|c|}{$\mathrm{q} 5(n=99)$} & \\
\hline & & & & & & & & & & & & & & & & & \\
\hline Total energy & (kcal) & 355 & \pm & 114 & 383 & \pm & 123 & 362 & \pm & 129 & 377 & \pm & 146 & 374 & \pm & 122 & 0.387 \\
\hline Energy from healthy foods * & (kcal) & 321 & \pm & 125 & 352 & \pm & 133 & 323 & \pm & 122 & 334 & \pm & 138 & 332 & \pm & 134 & 0.819 \\
\hline Energy from unhealthy foods & (kcal) & 35 & \pm & 81 & 31 & \pm & 61 & 39 & \pm & 81 & 43 & \pm & 97 & 42 & \pm & 107 & 0.362 \\
\hline \multicolumn{18}{|l|}{ Lunch } \\
\hline Total energy & (kcal) & 478 & \pm & 137 & 476 & \pm & 117 & 490 & \pm & 135 & 516 & \pm & 137 & 531 & \pm & 134 & 0.003 \\
\hline \multicolumn{18}{|l|}{ Dinner } \\
\hline Total energy & (kcal) & 538 & \pm & 169 & 627 & \pm & 214 & 562 & \pm & 195 & 560 & \pm & 178 & 609 & \pm & 178 & 0.152 \\
\hline Energy from healthy foods * & (kcal) & 530 & \pm & 168 & 611 & \pm & 205 & 551 & \pm & 194 & 552 & \pm & 176 & 597 & \pm & 177 & 0.153 \\
\hline Energy from unhealthy foods & (kcal) & 7 & \pm & 11 & 17 & \pm & 34 & 11 & \pm & 18 & 8 & \pm & 14 & 12 & \pm & 19 & 0.815 \\
\hline \multicolumn{18}{|l|}{ Between meal eating } \\
\hline Total energy & (kcal) & 208 & \pm & 159 & 178 & \pm & 129 & 213 & \pm & 165 & 210 & \pm & 143 & 194 & \pm & 152 & 0.951 \\
\hline Energy from healthy foods * & (kcal) & 52 & \pm & 96 & 40 & \pm & 70 & 41 & \pm & 80 & 70 & \pm & 93 & 66 & \pm & 125 & 0.071 \\
\hline Energy from unhealthy foods & (kcal) & 156 & \pm & 138 & 138 & \pm & 121 & 172 & \pm & 132 & 140 & \pm & 128 & 128 & \pm & 117 & 0.151 \\
\hline \multicolumn{18}{|l|}{ Daily } \\
\hline Total energy & (kcal) & 1579 & \pm & 321 & 1665 & \pm & 306 & 1628 & \pm & 369 & 1663 & \pm & 307 & 1708 & \pm & 315 & 0.013 \\
\hline Energy from healthy foods * & (kcal) & 1381 & \pm & 274 & 1479 & \pm & 282 & 1406 & \pm & 319 & 1472 & \pm & 294 & 1526 & \pm & 319 & 0.003 \\
\hline Energy from unhealthy foods & (kcal) & 198 & \pm & 177 & 185 & \pm & 140 & 222 & \pm & 161 & 191 & \pm & 156 & 182 & \pm & 170 & 0.548 \\
\hline Grain dishes & $(\mathrm{SV})$ & 2.90 & \pm & 0.85 & 2.87 & \pm & 0.76 & 2.83 & \pm & 0.76 & 2.99 & \pm & 0.85 & 3.11 & \pm & 1.06 & 0.055 \\
\hline Vegetable dishes & (SV) & 3.39 & \pm & 1.38 & 3.89 & \pm & 1.62 & 3.62 & \pm & 1.70 & 4.12 & \pm & 2.08 & 4.25 & \pm & 1.91 & 0.001 \\
\hline Fish and meat dishes & (SV) & 4.42 & \pm & 1.79 & 5.16 & \pm & 2.03 & 4.66 & \pm & 1.86 & 4.89 & \pm & 2.01 & 5.22 & \pm & 2.11 & 0.028 \\
\hline Milk and dairy products & (SV) & 3.67 & \pm & 1.41 & 4.06 & \pm & 2.23 & 3.72 & \pm & 1.92 & 3.96 & \pm & 1.81 & 3.84 & \pm & 2.06 & 0.643 \\
\hline Fruits & (SV) & 0.70 & \pm & 0.78 & 0.86 & \pm & 0.92 & 0.78 & \pm & 0.85 & 0.87 & \pm & 0.98 & 0.78 & \pm & 0.71 & 0.485 \\
\hline Confectionaries and sweetened beverages & $(\mathrm{SV})$ & 2.58 & \pm & 2.24 & 2.44 & \pm & 1.75 & 2.87 & \pm & 2.01 & 2.50 & \pm & 1.94 & 2.39 & \pm & 2.12 & 0.554 \\
\hline
\end{tabular}

Mean \pm SD, $p$ values are based on weighted one-way analysis of variance. NPFT, New Physical Fitness Test * Energy intake derived from grain dishes, vegetable dishes, fish and meat dishes, milk and dairy products, and fruit (based on the food groups defined by the Japanese Food Guide Spinning Top), as opposed to confectionaries and sweetened beverages.

Table 4. Dietary intake within New Physical Fitness Test score quintile groups among girls.

\begin{tabular}{|c|c|c|c|c|c|c|c|c|c|c|c|c|c|c|c|c|c|}
\hline \multirow{3}{*}{$\begin{array}{l}\text { Variables } \\
\text { Breakfast }\end{array}$} & & \multicolumn{15}{|c|}{ NPFT Score Quintile } & \multirow{2}{*}{$\begin{array}{l}p \text { for } \\
\text { Trend }\end{array}$} \\
\hline & & \multicolumn{3}{|c|}{$\mathrm{q} 1(n=70)$} & \multicolumn{3}{|c|}{$\mathrm{q} 2(n=96)$} & \multicolumn{3}{|c|}{$\mathrm{q} 3(n=82)$} & \multicolumn{3}{|c|}{$\mathrm{q} 4(n=91)$} & \multicolumn{3}{|c|}{ q5 $(n=92)$} & \\
\hline & & & & & & & & & & & & & & & & & \\
\hline Total energy & (kcal) & 317 & \pm & 119 & 323 & \pm & 125 & 328 & \pm & 107 & 353 & \pm & 124 & 355 & \pm & 105 & 0.008 \\
\hline Energy from healthy foods * & (kcal) & 289 & \pm & 133 & 286 & \pm & 123 & 294 & \pm & 125 & 333 & \pm & 129 & 311 & \pm & 118 & 0.030 \\
\hline Energy from unhealthy foods & (kcal) & 28 & \pm & 63 & 37 & \pm & 75 & 34 & \pm & 67 & 20 & \pm & 41 & 44 & \pm & 91 & 0.606 \\
\hline \multicolumn{18}{|l|}{ Lunch } \\
\hline Total energy & (kcal) & 417 & \pm & 126 & 480 & \pm & 127 & 448 & \pm & 110 & 470 & \pm & 115 & 520 & \pm & 138 & $<0.001$ \\
\hline \multicolumn{18}{|l|}{ Dinner } \\
\hline Total energy & (kcal) & 538 & \pm & 148 & 524 & \pm & 158 & 502 & \pm & 163 & 536 & \pm & 221 & 535 & \pm & 172 & 0.848 \\
\hline Energy from healthy foods * & (kcal) & 527 & \pm & 144 & 515 & \pm & 155 & 492 & \pm & 156 & 524 & \pm & 219 & 524 & \pm & 170 & 0.875 \\
\hline Energy from unhealthy foods & (kcal) & 10 & \pm & 18 & 10 & \pm & 13 & 10 & \pm & 18 & 13 & \pm & 25 & 10 & \pm & 13 & 0.515 \\
\hline \multicolumn{18}{|l|}{ Between meal eating } \\
\hline Total energy & (kcal) & 166 & \pm & 126 & 190 & \pm & 148 & 178 & \pm & 157 & 194 & \pm & 137 & 173 & \pm & 114 & 0.780 \\
\hline Energy from healthy foods * & (kcal) & 37 & \pm & 65 & 52 & \pm & 98 & 52 & \pm & 109 & 44 & \pm & 74 & 31 & \pm & 62 & 0.375 \\
\hline Energy from unhealthy foods & (kcal) & 129 & \pm & 110 & 138 & \pm & 124 & 126 & \pm & 125 & 150 & \pm & 123 & 142 & \pm & 104 & 0.338 \\
\hline \multicolumn{18}{|l|}{ Daily } \\
\hline Total energy & (kcal) & 1438 & \pm & 270 & 1517 & \pm & 278 & 1457 & \pm & 278 & 1554 & \pm & 329 & 1583 & \pm & 262 & 0.001 \\
\hline Energy from healthy foods * & (kcal) & 1271 & \pm & 265 & 1333 & \pm & 275 & 1287 & \pm & 264 & 1372 & \pm & 310 & 1387 & \pm & 251 & 0.005 \\
\hline Energy from unhealthy foods & (kcal) & 167 & \pm & 127 & 184 & \pm & 140 & 170 & \pm & 142 & 184 & \pm & 142 & 196 & \pm & 149 & 0.254 \\
\hline Grain dishes & $(\mathrm{SV})$ & 2.65 & \pm & 0.78 & 2.72 & \pm & 0.77 & 2.69 & \pm & 0.75 & 2.72 & \pm & 0.75 & 2.86 & \pm & 0.79 & 0.111 \\
\hline Vegetable dishes & (SV) & 3.63 & \pm & 1.48 & 3.92 & \pm & 1.59 & 3.61 & \pm & 1.63 & 3.85 & \pm & 1.60 & 4.13 & \pm & 1.65 & 0.101 \\
\hline Fish and meat dishes & (SV) & 4.06 & \pm & 1.52 & 4.28 & \pm & 1.65 & 4.37 & \pm & 2.11 & 4.66 & \pm & 1.99 & 4.54 & \pm & 1.69 & 0.037 \\
\hline Milk and dairy products & (SV) & 3.34 & \pm & 1.52 & 3.53 & \pm & 1.67 & 2.89 & \pm & 1.51 & 3.61 & \pm & 1.64 & 3.37 & \pm & 1.60 & 0.805 \\
\hline Fruits & $(\mathrm{SV})$ & 0.74 & \pm & 0.79 & 0.61 & \pm & 0.79 & 0.77 & \pm & 0.80 & 0.87 & \pm & 0.93 & 0.70 & \pm & 0.89 & 0.516 \\
\hline Confectionaries and sweetened beverages & $(\mathrm{SV})$ & 2.17 & \pm & 1.59 & 2.40 & \pm & 1.74 & 2.21 & \pm & 1.76 & 2.39 & \pm & 1.77 & 2.56 & \pm & 1.85 & 0.207 \\
\hline
\end{tabular}

Mean \pm SD, $p$ values are based on weighted one-way analysis of variance. NPFT, New Physical Fitness Test. * Energy intake derived from grain dishes, vegetable dishes, fish and meat dishes, milk and dairy products, and fruit (based on the food groups defined by the Japanese Food Guide Spinning Top), as opposed to confectionaries and sweetened beverages. 
In boys, significant positive correlations were observed with playing outside [standardized partial regression coefficient $(\beta)=0.132, p=0.005]$, after school exercise class ( $\beta=0.139, p=0.003)$, total daily energy intake $(\beta=0.151, p=0.001)$, and energy intake from healthy foods $(\beta=0.170, p<0.001$; Table 5$)$. In girls, significant positive correlations were observed with after school exercise class $(\beta=0.138, p=0.004)$, total daily energy intake $(\beta=0.176, p<0.001)$, and energy intake from healthy foods $(\beta=0.148, p=0.002$; Table 5). Multiple regression analysis was also conducted to assess the associations between NPFT scores and energy from healthy foods and unhealthy foods, and physical activity (Table 6). In boys, significant positive correlations were observed with energy intake from healthy foods $(\beta=0.151, p=0.001$; Model 1$)$, in addition to playing outside $(\beta=0.104, p=0.026)$ and after school exercise class $(\beta=0.126, p=0.008)$. Energy intake from healthy foods was significantly correlated $(\beta=0.120, p=0.011$; Model 3) even after including unhealthy food intake. Similarly, in girls, significant positive correlations were observed with energy intake from healthy foods $(\beta=0.141, p=0.004 ;$ Model 1$)$, in addition to after school exercise class $(\beta=0.132, p=0.006)$. Energy intake from healthy foods was also significantly correlated $(\beta=0.140, p=0.004$; Model 3) even after including for energy intake from unhealthy foods. Energy intake from unhealthy foods was not significantly correlated with NPFT scores in boys or girls.

Table 5. Linear regression analysis to investigate factors related to New Physical Fitness Test score.

\begin{tabular}{lcccc}
\hline \multicolumn{1}{c}{ Variables } & \multicolumn{2}{c}{ Boys } & \multicolumn{1}{c}{ Girls } \\
\cline { 2 - 5 } & $\boldsymbol{\beta}$ & $\boldsymbol{p}$ & $\boldsymbol{\beta}{ }^{*}$ & $\boldsymbol{p}$ \\
\hline Sleep duration & 0.019 & 0.684 & 0.002 & 0.967 \\
Playing outside & 0.132 & 0.005 & 0.082 & 0.088 \\
Studying at home & -0.059 & 0.210 & -0.021 & 0.667 \\
Sedentary activity at home & -0.007 & 0.877 & -0.006 & 0.895 \\
After school exercise class & 0.139 & 0.003 & 0.138 & 0.004 \\
After school classroom learning & 0.008 & 0.860 & 0.011 & 0.823 \\
After school music class & -0.013 & 0.775 & 0.079 & 0.104 \\
Eating breakfast every day & -0.013 & 0.774 & 0.003 & 0.944 \\
Daily total energy intake & 0.151 & 0.001 & 0.176 & $<0.001$ \\
Energy intake from healthy foods ${ }^{+}$ & 0.170 & $<0.001$ & 0.148 & 0.002 \\
Energy intake from unhealthy foods & -0.015 & 0.751 & 0.071 & 0.138
\end{tabular}

* Standardized regression coefficient adjusted for Rohrer's index. Sleep duration was classified into eight categories. The frequency of playtime outside was set as ' 1 ' for responses of "almost every day"; all other responses were set as dummy variables with a value of ' 0 '. Study time was classified into eight categories. Sedentary activity at home included total time spent watching television and participating in video gaming or reading comics, and was classified into four categories. A value of ' 1 ' was allocated for children engaged in after school exercise class, classroom learning, and/or music class, whereas children not engaged in after school classes including weekend classes were allocated dummy variables with a value of ' 0 '. A value of ' 1 ' was allocated for eating breakfast every day, whereas other responses were allocated dummy variables with a value of ' 0 '. ' Energy intake derived from grain dishes, vegetable dishes, fish and meat dishes, milk and dairy products, and fruits (based on the food groups defined by the Japanese Food Guide Spinning Top), as opposed to confectionaries and sweetened beverages. 
Table 6. Multiple regression analysis for New Physical Fitness Test score.

\begin{tabular}{lccccccc}
\hline \multirow{2}{*}{ Variables } & \multicolumn{2}{c}{ Model 1 } & \multicolumn{2}{c}{ Model 2 } & \multicolumn{2}{c}{ Model 3 } \\
\cline { 2 - 7 } & $\beta^{+}$ & $p$ & $\beta^{+}$ & $p$ & $\beta^{+}$ & $p$ \\
\hline Boys & & & & & & \\
$\quad$ Playing outside & 0.104 & 0.026 & 0.122 & 0.010 & 0.131 & 0.005 \\
$\quad$ After school exercise class & 0.126 & 0.008 & 0.137 & 0.004 & 0.133 & 0.005 \\
Energy from healthy foods * & 0.151 & 0.001 & - & - & 0.120 & 0.011 \\
$\quad$ Energy from unhealthy foods & - & - & -0.022 & 0.638 & -0.019 & 0.683 \\
\hline Girls & & & & & & \\
Playing outside & 0.082 & 0.088 & 0.088 & 0.070 & 0.088 & 0.066 \\
After school exercise class & 0.132 & 0.006 & 0.139 & 0.004 & 0.120 & 0.013 \\
Energy from healthy foods * & 0.141 & 0.004 & - & - & 0.140 & 0.004 \\
Energy from unhealthy foods & - & - & 0.063 & 0.195 & 0.076 & 0.116 \\
\hline
\end{tabular}

Each model was adjusted for Rohrer's index, sleep duration, study time, the sedentary activities at home, participation in after school classes (classroom learning and/or music), and eating breakfast every day. ${ }^{+}$Standardized regression coefficient. * Energy intake derived from grain dishes, vegetable dishes, fish and meat dishes, milk and dairy products, and fruits (based on the food groups defined by the Japanese Food Guide Spinning Top), as opposed to confectionaries and sweetened beverages.

\section{Discussion}

In the present study, NPFT scores were positively correlated with energy intake from healthy foods in children, in addition to physical activity.

Energy intake from healthy foods had a significant positive correlation, and in particular, higher intake of vegetable dishes in boys and higher intake of fish and meat dishes in both boys and girls were correlated with NPFT scores. Previous studies have reported higher grip and lower limb muscle strength in groups with higher protein intake [16], and positive correlations between motor fitness and frequency of fruit and vegetable consumption [7]. The consumption of energy from grains, vegetables, and fish and meat, rather than from confectionaries and sweetened beverages, promotes healthier bodies and provides the required energy and nutrients in children.

No significant correlation was observed between energy derived from unhealthy foods and NPFT scores. The Japanese Food Guide Spinning Top defines an adequate intake of sweets and sweetened beverages as $\leq 200 \mathrm{kcal}$ [15]. Means of energy intake from unhealthy foods for each of the five NPFT quintiles in the present study were all approximately $200 \mathrm{kcal}$. Although the quantitative effect of sweets and sweetened beverages is unknown, maintaining an appropriate level of intake for these food groups is important.

Participation in after school exercise class (an index of daily physical activity) was positively correlated with NPFT scores. Previous studies have reported that 13-year-old children who exercise three or more times per week have improved grip and 50-metre run [3], and that moderate-to-vigorous physical activity (associated with $\geq 3$ metabolic equivalents) by school-age and adolescent children is positively correlated with motor fitness $[7,17]$. Exercise lesson programmes such as baseball, soccer, and swimming often include basic skill practice in addition to competition. Thus, children attending exercise classes regularly participate in activities such as side-stepping and sit-ups, as well as endurance enhancing exercises such as jogging. The strength developed during these activities is then reflected in activities included in physical fitness tests and may be positively correlated with NPFT scores. The present study also found a significant positive correlation between playing outside at break time at school or after school with NPFT scores in boys; a positive tendency was observed also in girls. At the time of the survey, the participating children were allowed to play outside freely based on their own intention at break time. Reports have shown that children with three or more days per week of outside playtime (another index of daily physical activity) participate in significantly longer periods of light and total physical activity, as compared to children who do not play outside as frequently [18]. Higher frequencies of playing outside, corresponding to larger amounts of physical activity, affected NPFT scores. 
Recent studies have shown that inadequate physical activity could be influenced by screen time use among children [19]. This study did not observe significant correlations between NPFT scores and sedentary activities at home, including the total time spent watching television and participating in video gaming or reading comics. According to a recent review, evidence is weak and inconsistent with respect to the association between screen time use and fitness [20]. Further studies will be needed to clarify the association between motor fitness and screen time use in children.

This study did not observe significant correlations between NPFT scores and other lifestyle factors that previously suggested. Sandercock et al. [6] reported higher cardiorespiratory endurance values in adolescent children who ate breakfast every day. However, only $67.9 \%$ of the study participants in the same study [6] ate breakfast every day, as compared to approximately $98 \%$ of participants in the present study. The percentage of children who missed breakfast one or more days per week in the current study was approximately $2 \%$, which is a substantially smaller proportion than that observed previously. This difference may explain why the current study did not find breakfast consumption to be significantly correlated with NPFT scores. In the MEXT survey, children with eight or more hours of sleep per night had the highest NPFT scores [21]. However, previous studies that did not indicate correlations between sleep duration and motor fitness found mean sleep durations to be $9.6-9.7 \mathrm{~h}$ per night $[7,17]$. The present study found the mean sleep duration to be $9.6 \mathrm{~h}$ per night for both boys and girls, which supports the results of previous studies. Therefore, the lack of an observed correlation in this study may have been due to the children receiving adequate sleep.

The strength of this study was that it investigated the correlations between motor fitness and nutritional intake among approximately 900 primary school students. The use of dietary records allowed for consideration of the mutual effects of meal quantity and quality. An additional strength was that motor fitness was assessed from the multiple components of fitness using the NPFT according to the MEXT guidelines.

This study had several limitations. The present study was unable to include objective parameters of physical activities, such as actigraphy or pedometer use, due to the significant burden on participating teachers and children. Rather, the frequencies of playing outside and participating in after school exercise class were used. Because food intake was only surveyed for one day, the meals consumed on that day may not be indicative of children's habitual meals. However, participating children and guardians were asked to enter data into the dietary record of meals that children ate at home that most closely represented meals on an average day, and school lunches were provided in accordance with Japanese school lunch implementation standards [13] and thus represent an average daily meal. In addition, data on the guardians' educational backgrounds and household financial status could not be obtained. Finally, the response rate in this study was low at $44.6 \%$. The survey may thus have had a selection bias, with a significant number of respondents who had a high degree of involvement in the survey and a high level of interest in participating children's meals and health.

\section{Conclusions}

The present study found that energy from healthy foods is correlated with motor fitness in children in addition to their physical activity. This finding suggests that the consumption of healthy foods that include grains, vegetables, fruits, milk, and fish and meat, as well as exercise habits, are important for childhood motor fitness.

Supplementary Materials: The following supporting information can be downloaded at: https: / / www.mdpi.com/article/10.3390/ijerph19031819/s1, Brief summary of the New Physical Fitness Test Implementation Guideline. 
Author Contributions: Y.T., T.F., A.H. and Y.K. designed the research; Y.T., N.H., R.K. and T.H. conducted the research; Y.T., N.H. and K.I.-T. analyzed the data; Y.T., N.H. and K.I.-T. wrote the manuscript; Y.T. assumes primary responsibility for the final content. All authors have read and agreed to the published version of the manuscript.

Funding: This study was supported by Health and Labor Sciences Research Grant in 2019 for the 'Development of a food, nutrition, and dietary guide for healthy development in early childhood' (H29 Sukoyaka-Ippan 003). The funders (Ministry of Health, Labor and Welfare of Japan) had no role in the study design, data collection/analysis, decision to publish, or preparation of the manuscript.

Institutional Review Board Statement: This study was conducted according to the guidelines outlined in the Declaration of Helsinki. All procedures involving research study participants were approved by the Human Research Ethics Committees at Tokyo University of Agriculture (Reference numbers: 1122, 1130, 1501, 1605, 1704, 1802).

Informed Consent Statement: Written informed consent was obtained from all participants.

Data Availability Statement: The datasets used and/or analysed during the current study are available from the corresponding author on reasonable request.

Acknowledgments: We thank the elementary school principals and teachers for their support in conducting the present study.

Conflicts of Interest: The authors declare that they have no competing interests.

\section{References}

1. Ortega, F.B.; Ruiz, J.R.; Castillo, M.J.; Sjöström, M. Physical fitness in childhood and adolescence: A powerful marker of health. Int. J. Obes. 2008, 32, 1-11. [CrossRef]

2. García-Hermoso, A.; Ramírez-Campillo, R.; Izquierdo, M. Is Muscular Fitness Associated with Future Health Benefits in Children and Adolescents? A Systematic Review and Meta-Analysis of Longitudinal Studies. Sports Med. 2019, 49, 1079-1094. [CrossRef] [PubMed]

3. Ministry of Education, Culture, Sports, Science and Technology. Results of the FY2015 Survey on Physical Fitness and Motor Abilities. Available online: https://warp.ndl.go.jp/info:ndljp/pid/11293659/www.mext.go.jp/component/english/_icsFiles/ afieldfile/2017/01/25/1381545_002.pdf (accessed on 1 January 2022).

4. Tanaka, C.; Tremblay, M.S.; Okuda, M.; Tanaka, S. Association between 24-hour movement guidelines and physical fitness in children. Pediatr. Int. 2020, 62, 1381-1387. [CrossRef] [PubMed]

5. Chen, W.; Hammond-Bennett, A.; Hypnar, A.; Mason, S. Health-related physical fitness and physical activity in elementary school students. BMC Public Health 2018, 18, 195. [CrossRef] [PubMed]

6. Sandercock, G.R.H.; Voss, C.; Dye, L. Associations between habitual school-day breakfast consumption, body mass index, physical activity and cardiorespiratory fitness in English schoolchildren. Eur. J. Clin. Nutr. 2010, 64, 1086-1092. [CrossRef] [PubMed]

7. Zaqout, M.; Vyncke, K.; Moreno, L.A.; De Miguel-Etayo, P.; Lauria, F.; Molnar, D.; Lissner, L.; Hunsberger, M.; Veidebaum, T.; Tornaritis, M.; et al. Determinant factors of physical fitness in European children. Int. J. Public Health 2016, 61, 573-582. [CrossRef] [PubMed]

8. Ministry of Health, Labour and Wellfare. Dietary Reference Intakes for Japanese. 2015. Available online: https:/ / www.mhlw.go. jp/file/06-Seisakujouhou-10900000-Kenkoukyoku/Full_DRIs2015.pdf (accessed on 1 January 2022).

9. Emmett, P.M.; Jones, L.R. Diet, growth, and obesity development throughout childhood in the Avon Longitudinal Study of Parents and Children. Nutr. Rev. 2015, 73, 175-206. [CrossRef] [PubMed]

10. Jacka, F.N.; Rothon, C.; Taylor, S.; Berk, M.; Stansfeld, S.A. Diet quality and mental health problems in adolescents from East London: A prospective study. Soc. Psychiatry 2012, 48, 1297-1306. [CrossRef] [PubMed]

11. Lachat, C.; Hawwash, D.; Ocké, M.C.; Berg, C.; Forsum, E.; Hörnell, A.; Larsson, C.; Sonestedt, E.; Wirfält, E.; Åkesson, A.; et al. Strengthening the Reporting of Observational Studies in Epidemiology-Nutritional Epidemiology (STROBE-nut): An Extension of the STROBE Statement. PLOS Med. 2016, 13, e1002036. [CrossRef] [PubMed]

12. Ministry of Education, Culture, Sports, Science and Technology. Implementation Guidance of New Physical Fitness Test for Children Aged 6 to 11 Years. (In Japanese) Available online: http:/ / www.mext.go.jp/a_menu/sports/stamina/05030101/001.pdf (accessed on 1 January 2022).

13. Ministry of Education, Culture, Sports, Science and Technology. Japanese School Lunch Implementation Standards. (In Japanese) Available online: https://www.mext.go.jp/content/20201228-mxt_kenshoku-100003354_01.pdf (accessed on 1 January 2022).

14. Ministry of Education, Culture, Sports, Science and Technology. Standard Tables of Food Composition in Japan (Seventh Revised Version). 2015. Available online: https://www.mext.go.jp/en/policy/science_technology/policy/title01/detail01/1374030.htm (accessed on 1 January 2022). 
15. Yoshiike, N.; Hayashi, F.; Takemi, Y.; Mizoguchi, K.; Seino, F. A New Food Guide in Japan: The Japanese Food Guide Spinning Top. Nutr. Rev. 2008, 65, 149-154. [CrossRef] [PubMed]

16. Isanejad, M.; Mursu, J.; Sirola, J.; Kröger, H.; Rikkonen, T.; Tuppurainen, M.; Erkkilä, A.T. Dietary protein intake is associated with better physical function and muscle strength among elderly women. Br. J. Nutr. 2016, 115, 1281-1291. [CrossRef] [PubMed]

17. Carson, V.; Tremblay, M.S.; Chaput, J.-P.; Chastin, S.F. Associations between sleep duration, sedentary time, physical activity, and health indicators among Canadian children and youth using compositional analyses. Appl. Physiol. Nutr. Metab. 2016, 41, S294-S302. [CrossRef] [PubMed]

18. Schoeppe, S.; Duncan, M.J.; Badland, H.M.; Oliver, M.; Browne, M. Associations between children's independent mobility and physical activity. BMC Public Health 2014, 14, 91. [CrossRef] [PubMed]

19. Alotaibi, T.; Almuhanna, R.; Alhassan, J.; Alqadhib, E.; Mortada, E.; Alwhaibi, R. The Relationship between Technology Use and Physical Activity among Typically-Developing Children. Healthcare 2020, 8, 488. [CrossRef] [PubMed]

20. Stiglic, N.; Viner, R.M. Effects of screentime on the health and well-being of children and adolescents: A systematic review of reviews. BMJ Open 2019, 9, e023191. [CrossRef] [PubMed]

21. Ministry of Education, Culture, Sports, Science and Technology. The Handbook for Enhancement of the Physical Strength of Children. (In Japanese) Available online: http://www.mext.go.jp/a_menu/sports/kodomo/zencyo/1321132.htm (accessed on 23 April 2019). 